\title{
Pravastatin for Fetal Growth Restriction Associated with Preeclampsia
}

\author{
Kumasawa Keiichi*, Iriyama Takayuki, Nagamatsu Takeshi, Osuga Yutaka and Fujii Tomoyuki \\ Kumasawa Keiichi, Iriyama Takayuki, Nagamatsu Takeshi, Osuga Yutaka and Fujii Tomoyuki
}

Submission: December 06, 2018; Published: February 19, 2019

*Corresponding author: Keiichi Kumasawa MD, Ph.D, Department of Obstetrics and Gynecology, Faculty of Medicine, University of Tokyo, 3-1

Hongo, Bunkyo-ku, Tokyo, 113-8655, Japan

\begin{abstract}
Fetal growth restriction (FGR) is a serious condition that is strongly associated with fetal mortality and morbidity. Preeclampsia is one of the major causes of FGR. However, thus far, the only fundamental therapy for preeclampsia is termination of pregnancy, leading to preterm birth. Recently, statins were reported to improve regeneration of vascular endothelium, and pravastatin has attracted attention as a potential therapeutic candidate in the prevention of preeclampsia and related FGR. Pravastatin has been investigated in FGR model mice with preeclampsia, and successful results have been reported. In addition, a large volume of human data from pregnant women ingesting statins indicates the safety of pravastatin. Moreover, small clinical trials reported that pravastatin has strong effects on the prevention of FGR associated with preeclampsia. Pravastatin has the potential to improve the prognosis in pregnant women, fetuses, and neonates at risk of preeclampsia and FGR.
\end{abstract}

Keywords: Fetal growth restriction (FGR); preeclampsia; pravastatin; sFlt-1; PIGF

Abbreviations: FGR: Fetal Growth Restriction; FDA: Food and Drug Administration; PI: Pulsatility Index

\section{Introduction}

Fetal growth restriction (FGR) is a condition that affects $5-10 \%$ of pregnancies and is one of the most common causes of perinatal mortality and morbidity [1]. FGR may be classified as early or late depending on the time of diagnosis. Early FGR $(<32$ weeks $)$ is associated with critical alterations in placental implantation leading to increased hypoxia. Perinatal morbidity and mortality rates are high. Late FGR ( $\geq 32$ weeks) presents with slight deficiencies in placentation, leading to mild hypoxia; perinatal morbidity and mortality rates are relatively lower. There are many causes of FGR, including normal variant, chromosomal aberration, TORCH infection, umbilical cord abnormality, thrombophilia, preeclampsia, and others. Among them, preeclampsia affects approximately $5-10 \%$ of pregnant women. As preeclampsia is associated with placental dysfunction, it is often accompanied by FGR.

\section{Discussion}

\section{Preeclampsia and FGR}

Preeclampsia is one of the major problems in the perinatal period. Preeclampsia is a complex medical disorder; worldwide, it is responsible for $>500000$ fetal and neonatal deaths and $>70000$ maternal deaths each year [2]. Thus far, many studies have been conducted attempting to elucidate its pathogenesis and improve therapy. However, the fundamental treatment of preeclampsia is still the termination of pregnancy, which leads to an increase in preterm birth. Preterm birth is another major problem in obstetrics and neonatology. In 2017, Nicolides and colleagues reported that aspirin had a preventive effect on the onset of preeclampsia. By administering $150 \mathrm{mg}$ of aspirin to pregnant women in the highrisk group, the incidence of preeclampsia onset before 37 weeks was reduced by approximately $60 \%$ compared to the control group. However, aspirin did not improve the incidence of birth weight less than the $3 \mathrm{rd}$, 5th, and 10 th percentile compared with the placebo group [3].

\section{Statins}

Statins are drugs that lower the level of low-density lipoprotein cholesterol in the serum by inhibiting 3-hydroxy-3-methylglutarylcoenzyme reductase, which plays a critical role in the synthesis of endogenous cholesterol. Currently, six types of statins are used: natural (lovastatin, pravastatin, and simvastatin) and synthetic (atorvastatin, fluvastatin, and rosuvastatin). Recently, the pleiotropic effects of statins were reported [4]. The numerous cholesterol-independent effects of statins are associated with huge therapeutic potential. For example, angiogenesis is promoted 
at the sites of ischemia by improvement in the regeneration of vascular endothelium [5].

\section{FGR and preeclampsia model mice}

Among the six currently used statins, we chose pravastatin as a treatment for preeclampsia and its associated FGR. First, we created preeclampsia model mice. In this model, the placenta became smaller and the placenta showed impaired vasculogenesis. Furthermore, the model mice also developed FGR, which is often accompanied by preeclampsia. When we intraperitoneally administered pravastatin to this mouse model, in addition to improvement in hypertension, vasculogenesis of the placenta and FGR improved. In pravastatin-administered PE mice, serum sFlt1 level, a key factor in PE onset, declines and serum PIGF level, which is thought to be important for placenta formation, increases [6].

Experiments administering pravastatin to other preeclampsia model mice have also been reported by other groups. For example, systemic administration of adenoviral vectors expressing sFlt1 to mice resulted in preeclampsia [7]. Interestingly, pravastatin improved the vascular reactivity in this murine model of PE and decreased sFlt-1 levels [7]. Using this same model of PE induced by general overexpression of sFlt-1, other authors suggested that pravastatin's ability to prevent the preeclamptic phenotype may be mediated through pleiotropic mechanisms involving a prosurvival/antiapoptotic mitogen-activated protein kinase pathway in trophoblasts [8]. Saad AF, Costantine MM, and colleagues revealed that pravastatin treatment in sFlt-1 mice reduced serum sFlt-1 levels at day 18 to a level similar to that of the control group. Placental PIGF and VEGF expression were upregulated, and markers of hypoxia downregulated to levels similar to those of control group [9]. In the model, pravastatin improved serum levels of sFlt-1.

\section{Clinical research}

Despite progress in preeclampsia research and related FGR using animal models, clinical application of pravastatin in pregnant women has not progressed significantly. Because statins are classified as category $\mathrm{X}$ by the Food and Drug Administration (FDA), use of statins for pregnant women has been limited.

Over the previous ten years, reports on pregnant women taking pravastatin have accumulated. In addition, although pravastatin has been administered to a small number of high-risk pregnant women for several years, randomized controlled trials have not been reported. Statins were classified as category $\mathrm{X}$ by the FDA in 1979, therefore it has been difficult to use pravastatin for the prevention of pregnancy hypertension syndrome. However, based on accumulated data, pravastatin hasn't been shown to be teratogenic $[10,11]$. There are also reports that abnormalities have not increased among infants born from pregnant women who took pravastatin for reasons such as unknown pregnancy. Bateman and colleagues reported that, in a cohort study of 890000 people in the United States, approximately 1200 pregnant women ingested statins in the early pregnancy period, representing the period of organogenesis, and the incidence of teratogenicity did not increase [12].

Other groups have reported similar results $[13,14]$. Furthermore, in 2015, the FDA abolished the traditional category classification because it did not comply with the actual situation of drug use. Costantine and colleagues examined the dynamics of cholesterol in humans by consulting with the FDA and administering pravastatin at a dose of $10 \mathrm{mg} /$ day to a preeclampsia high-risk group. As a result, although the blood cholesterol concentration in the mother decreased with pravastatin administration, little difference was observed in the cholesterol concentration in the umbilical cord blood [15]. In this report, the occurrence of preeclampsia was 5 out of 10 patients in the placebo group versus 0 of out 10 patients in the placebo group. Birth weights were $2877 \mathrm{~g} \pm 630 \mathrm{~g}$ in the placebo group and $3018 \pm 260 \mathrm{~g}$ in the pravastatin group. The pravastatin group was associated with larger birth weight. Furthermore, this study reported that pravastatin administration lowered serum sFlt1 level and increased serum PlGF level. These results suggest that pravastatin contributed to maintenance and improvement of placental function. Furthermore, Girardi and colleagues showed that pravastatin was effective in cases with PE onset despite LDA + heparin administration in pregnant women with APS. The median number of gestational weeks until delivery in the LDA + heparin control group was 26.5 weeks, whereas in the pravastatin group it was extended to 36 weeks. The median birth weight was $900 \mathrm{~g}$ in the control group and $2390 \mathrm{~g}$ in the pravastatin group. In both the control group and the pravastatin group, the median birth weight was out of the FGR range at each gestational week. In the control group, the mean uterine artery pulsatility index (PI) was above the 95th percentile. Therefore, in the LDA + heparin group, if pregnancy continued, the fetus was expected to develop FGR and IUFD. In the pravastatin group, PI values decreased after pravastatin treatment.

\section{Conclusion}

Research on pravastatin for the prevention and treatment of preeclampsia and related FGR has been performed with animal models. Furthermore, its safety was verified by the accumulation of outcome data from pregnant women taking statins. Pharmacokinetic data of pravastatin has been examined with the administration of pravastatin to a small number of pregnant women at high risk of preeclampsia and FGR. Furthermore, prophylactic and therapeutic effects have been confirmed by administration of pravastatin to high risk pregnant women. It is expected that the timing and target of pravastatin administration will be studied in the future. Furthermore, large scale clinical studies will verify whether pravastatin will be a powerful prophylactic and therapeutic agent for preeclampsia and related FGR. Pravastatin has the potential to improve the prognosis of many pregnant women, fetuses, and neonates at risk of preeclampsia and FGR. 


\section{References}

1. Nardozza LM, Caetano AC, Zamarian AC, Mazzola JB, Silva CP, et al. (2017) Fetal growth restriction: current knowledge. Arch Gynecol Obstet 295(5): 1061-1077.

2. Brown MA, Magee LA, Kenny LC, Karumanchi SA, McCarthy FP, et al. (2018)International Society for the Study of Hypertension in, Hypertensive Disorders of Pregnancy: ISSHP Classification, Diagnosis, and Management Recommendations for International Practice Hypertension: 72(1): 24-43.

3. Rolnik DL, Wright D, Poon LC, O'Gorman N, Syngelaki A, et al. (2017) Nicolaides, Aspirin versus Placebo in Pregnancies at High Risk for Preterm Preeclampsia. N Engl J Med 377(7): 613-622.

4. Wang CY, Liu PY, Liao JK, et al. (2008) Pleiotropic effects of statin therapy: molecular mechanisms and clinical results. Trends Mol Med 14(1): 37-44.

5. Ray KK, Cannon CP, et al. (2005) The potential relevance of the multiple lipid-independent (pleiotropic) effects of statins in the management of acute coronary syndromes. J Am Coll Cardiol 46(8): 1425-1433.

6. Kumasawa K, Ikawa M, Kidoya H, Hasuwa, Saito Fujita T, et al. (2011) Pravastatin induces placental growth factor (PGF) and ameliorates preeclampsia in a mouse model. Proc Natl Acad Sci USA 108(4): 14511455.

7. Costantine MM, Tamayo E, Lu E, Bytautiene E, Longo M, et al. (2010) Using pravastatin to improve the vascular reactivity in a mouse model of soluble fms-like tyrosine kinase-1-induced preeclampsia. Obstet Gynecol 116(1): 114-120.
8. Saad AF, Diken ZM, Kechichian TB, Clark SM, Olson GL, et al. (2016) Pravastatin Effects on Placental Prosurvival Molecular Pathways in a Mouse Model of Preeclampsia. Reprod Sci 23(11): 1593-1599.

9. Saad AF, Kechichian T, Yin H, Sbrana E, Longo M, et al. (2014) Effects of pravastatin on angiogenic and placental hypoxic imbalance in a mouse model of preeclampsia. Reprod Sci 21(1): 138-145.

10. Karalis DG, Hill AN, Clifton S, Wild RA, et al. (2016) The risks of statin use in pregnancy: A systematic review: J Clin Lipidol 10(5)1081-1090.

11. Zarek J, Koren G (2014) The fetal safety of statins: a systematic review and meta-analysis, J Obstet Gynaecol Can 36(6): 506-509.

12. Bateman BT, Hernandez Diaz S, Fischer MA, Seely EW, Ecker JL, et al. (2015) Huybrechts, Statins and congenital malformations: cohort study. BMJ 350: 1035.

13. Lecarpentier E, Morel O, Fournier T, Elefant E, Chavatte Palmer P, et al (2012) Statins and pregnancy: between supposed risks and theoretical benefits. Drugs 72(6): 773-788.

14. Winterfeld U, Allignol A, Panchaud A, Rothuizen LE, Merlob P, et al. (2013) Pregnancy outcome following maternal exposure to statins: a multicentre prospective study. BJOG 120(4): 463-471.

15. Costantine MM, Cleary K, Hebert MF, Ahmed MS, Brown LM, et al (2016) Eunice Kennedy Shriver National Institute of Child, N. Human Development Obstetric-Fetal Pharmacology Research Units, Safety and pharmacokinetics of pravastatin used for the prevention of preeclampsia in high-risk pregnant women: a pilot randomized controlled trial. Am J Obstet Gynecol 214: 720e721-720e717.

\section{Your next submission with Juniper Publishers will reach you the below assets}

- Quality Editorial service

- Swift Peer Review

- Reprints availability

- E-prints Service

- Manuscript Podcast for convenient understanding

- Global attainment for your research

- Manuscript accessibility in different formats

( Pdf, E-pub, Full Text, Audio)

- Unceasing customer service

Track the below URL for one-step submission https://juniperpublishers.com/online-submission.php 